\title{
Interposed Free Bone Fragment in Dislocated Medial Epicondyle Fracture of the Elbow: A Case Report
}

\author{
Yosuke Kaneko $^{\text {a }}$, Kensuke Ochia, b, c, d, Yukio Horiuchi ${ }^{\mathrm{a}}$
}

\begin{abstract}
A 13-year-old girl fell down on her left hand, resulting in elbow dislocation with medial epicondyle fracture of the humerus. The dislocation was immediately reduced manually; however, open reduction and internal fixation of the fracture was performed because the medial epicondyle showed $>5 \mathrm{~mm}$ displacement. Her passive motion under general anesthesia was smooth and full after the fixation. However, her elbow motion was limited to $60^{\circ}$ postoperatively. Postoperative imaging showed a free bone fragment interposed within her elbow. We removed the interposed bone fragment, and the patient showed full active elbow motion without pain in 4 weeks. To the best of our knowledge, there is few report of elbow fracture dislocation with an interposed free bone fragment in medial epicondyle fracture. The possibility of small isolated bone fragment interposition should be considered in medial epicondyle fractures with elbow dislocation.
\end{abstract}

Keywords: Bone fragment; Medial epicondyle fracture; Dislocation; Interposed; Humerus; Elbow

\section{Introduction}

Medial epicondyle fracture of the humerus is one of the most common elbow fractures in children [1-5]; however, to our knowledge, there is few report of elbow fracture dislocation with an interposed free bone fragment in medial epicondyle

\footnotetext{
Manuscript accepted for publication May 19, 2014

a Department of Orthopedic Surgery, Kawasaki Municipal Kawasaki Hospital, 12-1 Shinkawa-dori, Kawasaki-ku, Kawasaki, Kanagawa, 210-0013, Japan

${ }^{b}$ Department of Orthopedic Surgery, Institute of Rheumatology, Tokyo Women's Medical University, Japan

cDepartment of Orthopaedic Surgery, School of Medicine, Keio University, Tokyo, Japan

${ }^{\mathrm{d} C}$ Corresponding Author: Kensuke Ochi, Department of Orthopaedic Surgery, School of Medicine, Keio University, 35 Shinanomachi, Shinjuku, Tokyo, 160-0016, Japan.

Email: ochi@1998.jukuin.keio.ac.jp
}

doi: http://dx.doi.org/10.14740/jmc1819w fracture [6]. The purpose of this report is to show a case of such condition, and to show how to diagnose and treat it.

\section{Case Report}

A 13-year-old girl fell down on her left hand. Her general physician diagnosed her injury as left elbow dislocation with medial epicondyle fracture of the humerus (Fig. 1). The physician casted her elbow after manual reduction, and referred her to us for surgical evaluation. Preoperative radiography showed successful elbow dislocation reduction; however, the medial epicondyle fragment was displaced by $>5 \mathrm{~mm}$. We performed open reduction and internal fixation of the fracture under general anesthesia. Her bone fragment with flexors adapted well with the fractured site of her humerus, and was fixed by tension-band wiring. Passive motion of her elbow was smooth and full after fixation (Fig. 2). However, her elbow motion was limited to $60^{\circ} 5$ weeks postoperatively. Adaptation of her elbow joint was poor on radiography, and computed tomography showed an interposed bone fragment within her elbow (Fig. 3). The next week, an ad-

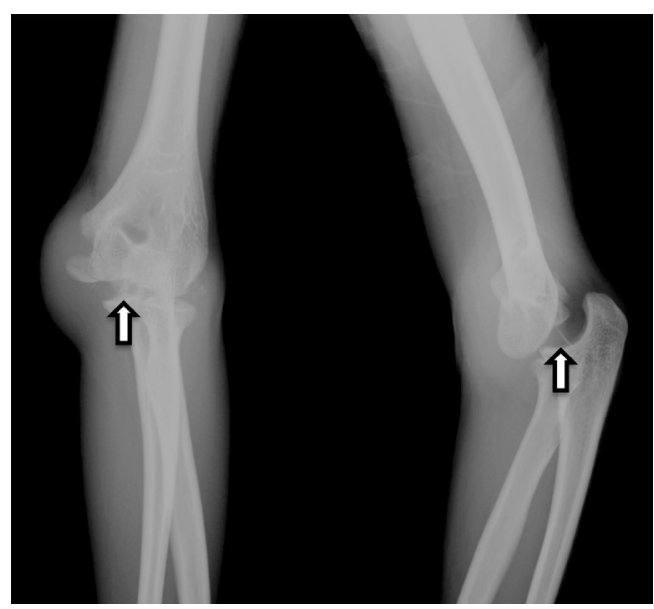

Figure 1. Radiograph obtained soon after the patient's visit to the general physician (Watson-Jones type 3). Careful retrospective evaluation showed a free interposed bone fragment within the elbow (arrow). 




Figure 2. Radiograph obtained after the primary operation. Careful retrospective evaluation showed a free interposed bone fragment within the elbow (arrow)

ditional operation was performed to remove the interposed bone fragment (Fig. 4) and wires. She had full elbow motion without pain 10 weeks after the primary operation.

\section{Discussion}

Medial epicondyle fractures are common in children, and elbow dislocation accompanying bone fragment interposition with flexors is reported to complicate more than $5 \%$ of such cases [1]. However, to our knowledge, few reports of elbow dislocation and medial epicondyle fracture with an interposed small free bone fragment exist [6].

The mechanism underlying small free bone fragment interposition is unclear. One of the possibilities in this case could be the following: the medial epicondyle fragment with flexors was impacted in her joint at the time of injury. This fragment contained the growth plate (Salter-Harris type 2). The interposed fragment with flexors was immediately pulled out from the joint by the power of the attached flexors. However, a small bony fragment proximal to the growth plate detached from the original fragment during this course, and remained within the joint. External force could also detach the fragment if the patient fell directly on her elbow.

We did not recognize the interposed free bone fragment, and simply fixed the fragment with flexors in the primary operation. To prevent such a case, we recommend the following. Surgeons should always be aware of the possibility of small free bony fragment interposition. Careful sequential evaluation of radiographs or computed tomography images could suggest the presence of an interposed fragment (Fig. 4). Next, passive motion of the elbow joint under general anesthesia may not be of use to confirm good joint surface congruity. Direct observation of the joint space is necessary to confirm that nothing is interposed within the joint.

\section{Acknowledgement}

We thank Professor Y. Toyama and other members of the Department of Orthopedic Surgery, Keio University School of Medicine for their support. We also thank Professor S. Mo-

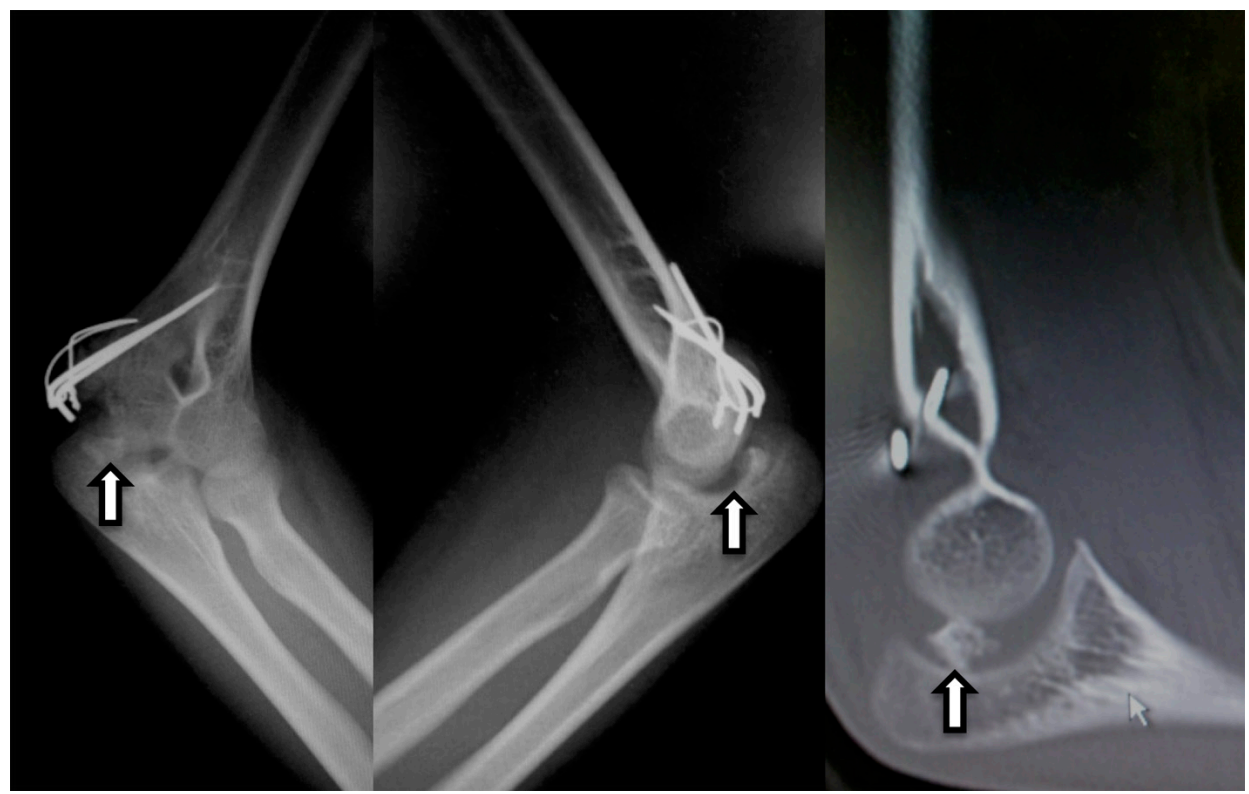

Figure 3. Radiograph (left) and computed tomography imaging (right) 5 weeks after the primary operation. Note that congruity of the joint was abnormal on the radiograph. Computed tomography clearly showed the interposed bone fragment. 


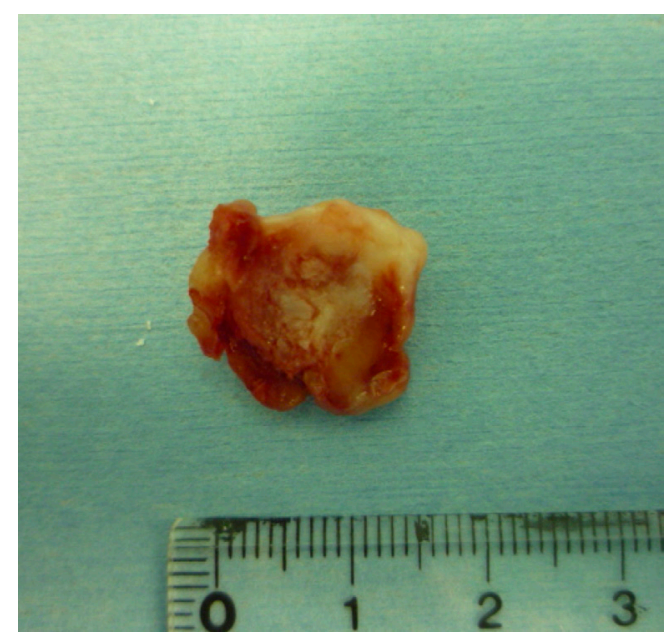

Figure 4. Removed free bone fragment. Neither cartilage nor muscle was observed in this $15 \times 15 \mathrm{~mm}$ fragment.

mohara and other members of the Institute of Rheumatology, Tokyo Women's Medical University for their support.

\section{Grant Support}

This work was supported in part by the Academic Research Project Grant from the Japanese Society for Surgery of the Hand, the Japan Society for the Promotion of Science, the Nakatomi Foundation, the Japan Orthopedics and Traumatology Foundation and Japan Osteoporosis Foundation to
KO.

\section{Conflict of Interest}

None declared.

\section{References}

1. Blount WP. Injuries about the elbow. Fractures in children. Baltimore: Williams \& Wilkins; 1955. p. 26-75.

2. Josefsson PO, Danielsson LG. Epicondylar elbow fracture in children. 35-year follow-up of 56 unreduced cases. Acta Orthop Scand. 1986;57(4):313-315.

3. Lee HH, Shen HC, Chang JH, Lee CH, Wu SS. Operative treatment of displaced medial epicondyle fractures in children and adolescents. J Shoulder Elbow Surg. 2005;14(2):178-185.

4. Louahem DM, Bourelle S, Buscayret F, Mazeau P, Kelly P, Dimeglio A, Cottalorda J. Displaced medial epicondyle fractures of the humerus: surgical treatment and results. A report of 139 cases. Arch Orthop Trauma Surg. 2010;130(5):649-655.

5. Patel NM, Ganley TJ. Medial epicondyle fractures of the humerus: how to evaluate and when to operate. J Pediatr Orthop. 2012;32(Suppl 1):S10-13.

6. Fowles JV, Slimane N, Kassab MT. Elbow dislocation with avulsion of the medial humeral epicondyle. J Bone Joint Surg Br. 1990;72(1):102-104. 